\title{
Effects of Short Term Metformin Treatment on Brown Adipose Tissue Activity and Plasma Irisin Levels in Women with Polycystic Ovary Syndrome: A Randomized Controlled Trial
}

Authors

Flávia R. Oliveira1, 6 , Marcelo Mamede², Mariana F. Bizzi ${ }^{3}$, Ana Luiza L. Rocha¹, Cláudia N. Ferreira ${ }^{4}$, Karina B. Gomes ${ }^{5}$ (D), Ana L. Cândido ${ }^{3}$, Fernando M. Reis ${ }^{1}$ (D)

Affiliations

1 Department of Obstetrics and Gynecology, Universidade Federal de Minas Gerais, Belo Horizonte, Brazil

2 Department of Anatomy and Imaging, Universidade Federal de Minas Gerais, Belo Horizonte, Brazil

3 Department of Internal Medicine, Universidade Federal de Minas Gerais, Belo Horizonte, Brazil

4 Technical College, Universidade Federal de Minas Gerais, Belo Horizonte, Brazil

5 Clinical and Toxicological Analyses, Universidade Federal de Minas Gerais, Belo Horizonte, Brazil

6 Odete Valadares Maternity Hospital, Fundação Hospitalar do Estado de Minas Gerais, Belo Horizonte, Brazil

Key words

brown adipose tissue, PET-CT, PCOS, metformin, irisin, randomized controlled trial

received $\quad 14.01 .2020$

accepted after revision $\quad 06.04 .2020$

published online 04.05.2020

Bibliography

Horm Metab Res 2020; 52: 718-723

DOI 10.1055/a-1157-0615

ISSN 0018-5043

(c) 2020. Thieme. All rights reserved.

Georg Thieme Verlag KG, Rüdigerstraße 14,

70469 Stuttgart, Germany

Correspondence

Fernando M. Reis MD, PhD

Division of Human Reproduction, Department of Ob/Gyn

Hospital das Clínicas, Universidade Federal de Minas Gerais

Av. Alfredo Balena, $110,9^{\circ}$ andar

30130-100 Belo Horizonte

MG

Brazil

Tel.: + 55313307 9485, Fax: + 553133079299

fmreis@ufmg.br
Supplementary material is available under

https://doi.org/10.1055/a-1157-0615

\section{ABSTRACT}

Polycystic ovary syndrome (PCOS) is a chronic dysfunction associated with obesity and metabolic disorders that can be ameliorated by treatment with metformin. Brown adipose tissue (BAT) has been recently identified in adult humans, and irisin is a myokine that induces BAT formation. The aim of this randomized controlled trial was to evaluate whether a short term treatment with metformin alters BAT activity and plasma irisin levels in women with PCOS. The participants were randomly assigned to receive metformin ( $1500 \mathrm{mg} /$ day, $\mathrm{n}=21$ ) or placebo $(n=24)$ during 60 days. BAT activity was assessed by ${ }^{18} \mathrm{~F}$-FDG positron emission tomography-computed tomography (PET-CT) and plasma irisin levels were measured by enzyme immunoassay. The groups were similar in age, body measures, metabolic profile and PCOS phenotypes. BAT activity did not change significantly in the women treated with metformin (median $\Delta$ SUV $_{\max }=-0.06 \mathrm{~g} / \mathrm{ml}$, interquartile interval -2.81 to $0.24 \mathrm{~g} / \mathrm{ml}, \mathrm{p}=0.484$, Wilcoxon's test) or placebo (median $\Delta S U V_{\max }=0.98 \mathrm{~g} / \mathrm{ml}$, interquartile interval -2.94 to 4.60 $\mathrm{g} / \mathrm{ml}, \mathrm{p}=0.386$ ). In addition, plasma irisin levels remained unchanged in the groups treated with metformin (median $\Delta=-98$ $\mathrm{ng} / \mathrm{ml}$, interquartile interval -366 to $60 \mathrm{ng} / \mathrm{ml}, \mathrm{p}=0.310$ ) and placebo (median $\Delta=28 \mathrm{ng} / \mathrm{ml}$, interquartile interval -1260 to $215 \mathrm{ng} / \mathrm{ml}, \mathrm{p}=0.650$ ). These results suggest that in PCOS women BAT activity and plasma irisin levels may not change after a brief treatment with metformin. 


\section{Introduction}

Polycystic ovary syndrome (PCOS) is an endocrine disorder affecting at least one in ten adult women and characterized by androgen excess, ovulatory dysfunction and insulin resistance, which manifest as a variety of symptoms over a spectrum of clinical phenotypes $[1,2]$. Although insulin resistance and glucose intolerance are not diagnostic criteria of PCOS, these features are very frequent and contribute to part of the morbidity associated with the syndrome [3].

Brown adipose tissue (BAT) is a highly energetic tissue with a unique mitochondrial expression of uncoupling protein-1 (UCP-1) that converts the cell respiration electrochemical gradient into heat, using glucose and fatty acids as substrate [4-6]. The presence of BAT in humans can be traced by positron emission tomography (PET) with ${ }^{18} \mathrm{~F}$-fluorodeoxyglucose ( $\left.{ }^{18} \mathrm{~F}-\mathrm{FDG}\right)$, a functional imaging method that evaluates areas of high metabolic activity [7]. BAT cells are found mainly in the supraclavicular, cervical, and paraesternal regions [8] and BAT activity is reduced in older and overweight people $[6,7]$. In women with PCOS, we observed lower BAT activity in the thoracic region when compare to control women of similar body mass index (BMI) but smaller waist circumference [9].

The mechanisms behind insulin resistance in women with PCOS include insulin receptor signaling defects and inhibition of insulin-mediated glucose uptake in adipocytes [10]. Metformin, a biguanide that has been used for PCOS treatment since 1994, is able to revert most of the metabolic abnormalities of PCOS $[11,12]$. By increasing insulin sensitivity, metformin treatment reduces ovarian androgen production and can restore ovulation in PCOS women [12]. Another possible action mechanism of metformin could be inducing transdifferentiation of white adipocytes into BAT $[13,14]$ through increased production of irisin, a myokine that can induce UCP-1 activation and BAT formation [15].

Thus, the aim of this randomized, double-blind, placebo-controlled trial was to evaluate whether a short term treatment with metformin alters BAT activity and plasma irisin levels in women with PCOS.

\section{Subjects and Methods}

\section{Study approval and registration}

This randomized controlled trial (RCT) was approved by the Research Ethics Committee of Universidade Federal de Minas Gerais (Decision Number 389.782) and registered at the Brazilian Clinical Trials Registry, REBEC (Primary ID Number RBR-47tvky) and Brazilian Ministry of Health Human Research Registry (Protocol ID 17127713.2.0000.5149). All patients included in this study signed an informed consent.

\section{Participants}

The study population consisted of 45 PCOS women aged 18-45 years, enrolled from April 2015 to September 2017 at the outpatient facilities of two teaching hospitals in Belo Horizonte, Brazil. The diagnosis of PCOS was made according to the 2003 Rotterdam ESHRE/ASRM PCOS Consensus Workshop Group diagnostic criteria [16]. We excluded patients with hypo or hyperthyroidism, hyperprolactinemia, 21-hydroxylase deficiency, Cushing's syndrome and androgen producing tumors. We also excluded patients who had used anti-androgen drugs in the last 6 months or any hormones or metformin in the last 2 months, pregnant or puerperal women, women who were breastfeeding, and patients with diabetes or oral glucose intolerance.

Body measurements were performed by the same professional with the participants wearing lightweight clothing without shoes. Physical activity was assessed through the International Physical Activity Questionnaire, quantified in min/week and classified into very active, active or sedentary as detailed elsewhere $[17,18]$. Women were classified as alcohol drinkers if they reported any quantity of alcohol consumption in the past month and as smokers if they smoked at least one cigarette per day $[19,20]$.

\section{Brown adipose tissue activity measurement}

BAT activity was measured using ${ }^{18} \mathrm{~F}$-FDG PET-CT with cold activation in the morning after $9-12 \mathrm{~h}$ fasting, as previously described [9]. After 1 hour resting at $19{ }^{\circ} \mathrm{C},{ }^{18} \mathrm{~F}-\mathrm{FDG}(0.1 \mathrm{mCi} / \mathrm{kg})$ was administered intravenously and the subjects remained in the same cold conditions for another hour. Afterwards, a whole-body PET-CT scan was performed at $20^{\circ} \mathrm{C}$ using a dedicated PET-CT system (Discovery PET-CT 600, GE Healthcare, Milwaukee, EUA). Low-dose CT without contrast enhancement was performed first and used for attenuation correction and anatomic image fusion [90 kV, 10-120 $\mathrm{mAs}$ (smart $\mathrm{mA}$ ) with $3.75 \mathrm{~mm}$ section thickness]. The PET images were reconstructed in a $192 \times 192$ matrix, using an Ordered Subsets Expectation Maximization (OSEM)-like algorithm, with 2 iterations and 24 subsets.

${ }^{18} \mathrm{~F}$-FDG uptake in fat areas identified by CT was quantified using a dedicated workstation (Xeleris, GE Healthcare) [21]. BAT activity was expressed in $\mathrm{g} / \mathrm{ml}$ as the maximum standardized uptake value $\left(S U V_{\text {max }}\right)$, defined as the tissue radioactivity concentration $(\mathrm{KBq} /$ $\mathrm{ml}$ ) divided by the injection dose per lean body mass (KBq/g). In each one of four body regions (cervico-thoracic, axillary, thoracic, and abdominal) a region of interest (ROI) was delimited around the most dense uptake zone and used to calculate the SUV $\max$. The highest BAT uptake value was used as the total BAT activity. All images were evaluated by two independent readers (experienced nuclear physician and radiologist), blinded to the clinical findings and laboratorial results.

\section{Irisin assay}

Blood samples were collected at 9:00 AM after an 8-hour fast between the $3^{\text {rd }}$ and $5^{\text {th }}$ days of menstrual cycle, and processed as described elsewhere [9]. Plasma irisin levels were assayed using a commercial ELISA kit (CUSABIO, catalog number CSB-EQ027943HU), following strictly the manufacturer's instructions, in duplicate and with the operator blinded to the patient group. The intra-assay and inter-assay coefficients of variation, using three samples of known concentration, were $<8$ and $<10 \%$, respectively. The assay sensitivity was $0.78 \mathrm{ng} / \mathrm{ml}$.

\section{Intervention}

The participants were randomly assigned to receive 60 days of treatment with either metformin (Glifage XR, Merck, $3 \times 500 \mathrm{mg}$, single daily dose at night, $n=21)$, or placebo tablets $(n=24)$. The treatment duration and dose were chosen because a systematic review showed that 8 weeks (56 days) and $1500 \mathrm{mg} /$ day were the minimum required to achieve metabolic improvement in women 
with PCOS [22]. Randomization was performed through a computer generated random number list. The personnel involved in participant enrollment received sequentially numbered, opaque, sealed flasks containing the metformin or placebo tablets.

The intervention period ranged from April 2015 to September 2017 and the last follow-up assessment was in September 2017. The main outcome measure was the change in total BAT level, and a secondary outcome was the change in plasma irisin level.

\section{Statistical analysis}

All participants available to follow-up were included in the outcome analyses regardless of adherence to the treatment protocol (modified intention-to-treat principle) [23]. Continuous variables were tested for normal distribution by Shapiro-Wilk test. Normally distributed variables were expressed as means \pm SD and compared between groups using unpaired Student's $t$-test. Non-normal variables were expressed as medians with quartiles and compared by Mann-Whitney U-test (between groups) or Wilcoxon matchedpairs signed rank test (post-treatment vs. pre-treatment). Categorical variables were submitted to Chi-square test with continuity (Yates) correction or Fisher's exact test, as appropriate. All tests were two-tailed and $p<0.05$ was considered statistically significant. Sample size calculation indicated that 21 participants per group would be sufficient to detect a minimum difference of $6 \mathrm{~g} / \mathrm{ml}$ in the change of BAT activity ( $\triangle \mathrm{SUV}_{\max }$ ) between the two treatment groups with $95 \%$ confidence and $80 \%$ statistical power.

- Table 1 Baseline characteristics of the study participants.

\begin{tabular}{|c|c|c|c|}
\hline Variable & Metformin $(n=21)$ & Placebo $(n=24)$ & p-Value \\
\hline Age (years) & $30.3 \pm 4.5$ & $30.9 \pm 6.4$ & 0.641 \\
\hline $\begin{array}{l}\text { Weight }(\mathrm{kg}) \\
\text { Height }(\mathrm{cm}) \\
\text { Body mass index }\left(\mathrm{kg} / \mathrm{m}^{2}\right)\end{array}$ & $\begin{array}{l}73.2 \pm 12.7 \\
161.4 \pm 6.5 \\
28.1 \pm 4.3\end{array}$ & $\begin{array}{l}73.5 \pm 13.7 \\
160.9 \pm 6.0 \\
28.3 \pm 4.9\end{array}$ & $\begin{array}{l}0.873 \\
0.707 \\
0.900\end{array}$ \\
\hline Waist circumference (cm) & $91.2 \pm 10.8$ & $90.7 \pm 13.9$ & 0.932 \\
\hline $\begin{array}{l}\text { Blood Pressure }(\mathrm{mmHg}) \\
\text { Systolic } \\
\text { Diastolic }\end{array}$ & $\begin{array}{l}110(110-120) \\
70(70-80)\end{array}$ & $\begin{array}{l}120(103-120) \\
75(63-80)\end{array}$ & $\begin{array}{l}0.230 \\
0.951\end{array}$ \\
\hline Smoking (n, \%) & $2(10 \%)$ & $4(17 \%)$ & 0.670 \\
\hline Alcohol drinking $(\mathrm{n}, \%)$ & $4(19 \%)$ & $6(25 \%)$ & 0.729 \\
\hline $\begin{array}{l}\text { Physical activity duration (min/week) } \\
\text { Physical activity classification ( } \mathrm{n}, \%) \\
\text { Very active } \\
\text { Active } \\
\text { Sedentary }\end{array}$ & $\begin{array}{l}360(165-615) \\
4(19 \%) \\
11(52 \%) \\
6(29 \%)\end{array}$ & $\begin{array}{l}460(188-715) \\
4(17 \%) \\
16(67 \%) \\
4(17 \%)\end{array}$ & $\begin{array}{l}0.495 \\
0.617\end{array}$ \\
\hline Current hypocaloric diet (n, \%) & $5(24 \%)$ & $2(10 \%)$ & 0.410 \\
\hline $\begin{array}{l}\text { Serum cholesterol }(\mathrm{mg} / \mathrm{dl}) \\
\text { Total } \\
\text { HDL-c }\end{array}$ & $\begin{array}{l}181.5 \pm 44.6 \\
48.4 \pm 13.9\end{array}$ & $\begin{array}{l}194.5 \pm 32.5 \\
46.7 \pm 11.3\end{array}$ & $\begin{array}{l}0.260 \\
0.622\end{array}$ \\
\hline Triglycerides (mg/dl) & $95(63-133)$ & $123(84-167)$ & 0.171 \\
\hline LAP index & $41(20-55)$ & $53(17-70)$ & 0.469 \\
\hline Fasting glucose (mg/dl) & $88.9 \pm 7.9$ & $87.7 \pm 6.5$ & 0.962 \\
\hline Serum insulin $(\mu \mathrm{lU} / \mathrm{ml})$ & $13.7 \pm 7.9$ & $14.6 \pm 10.7$ & 0.724 \\
\hline Serum TSH ( $\mu \mathrm{IU} / \mathrm{ml})$ & $2.02 \pm 0.81$ & $3.07 \pm 3.34$ & 0.890 \\
\hline $\begin{array}{l}\text { PCOS phenotype }(n, \%) \\
\text { Hyperandrogenism + anovulation + PCO } \\
\text { Anovulation + PCO } \\
\text { Hyperandrogenism + PCO }\end{array}$ & $\begin{array}{l}15(71 \%) \\
5(24 \%) \\
1(5 \%)\end{array}$ & $\begin{array}{l}11(46 \%) \\
12(50 \%) \\
1(4 \%)\end{array}$ & 0.237 \\
\hline Infertility (n, \%) & $20(95 \%)$ & $17(74 \%)$ & 0.097 \\
\hline Gravidity & $0(0-1)$ & $0(0-1)$ & 0.820 \\
\hline Previous metformin use (n, \%) & $10(48 \%)$ & $6(26 \%)$ & 0.242 \\
\hline Serum total testosterone $(\mathrm{ng} / \mathrm{dl})$ & $49.5 \pm 29.9$ & $48.4 \pm 29.6$ & 0.606 \\
\hline Serum prolactin (ng/ml) & $13.7 \pm 4.3$ & $13.9 \pm 4.2$ & 0.820 \\
\hline BAT activity (FDG uptake, SUV ${ }_{\max }$ ) & $7.4(0.9-16.4)$ & $5.2(0.9-14.4)$ & 0.946 \\
\hline Plasma irisin (ng/ml) & 710 (335-1099) & 950 (424-2152) & 0.195 \\
\hline
\end{tabular}

Data are expressed as means $\pm S D$ or medians (interquartile ranges). $p$-Values refer to unpaired $t$-test, Mann-Whitney test, Chi-square test, or Fisher's exact test, as appropriate. LAP: Lipid accumulation product; PCO: Polycystic and/or enlarged ovaries in ultrasound imaging. 


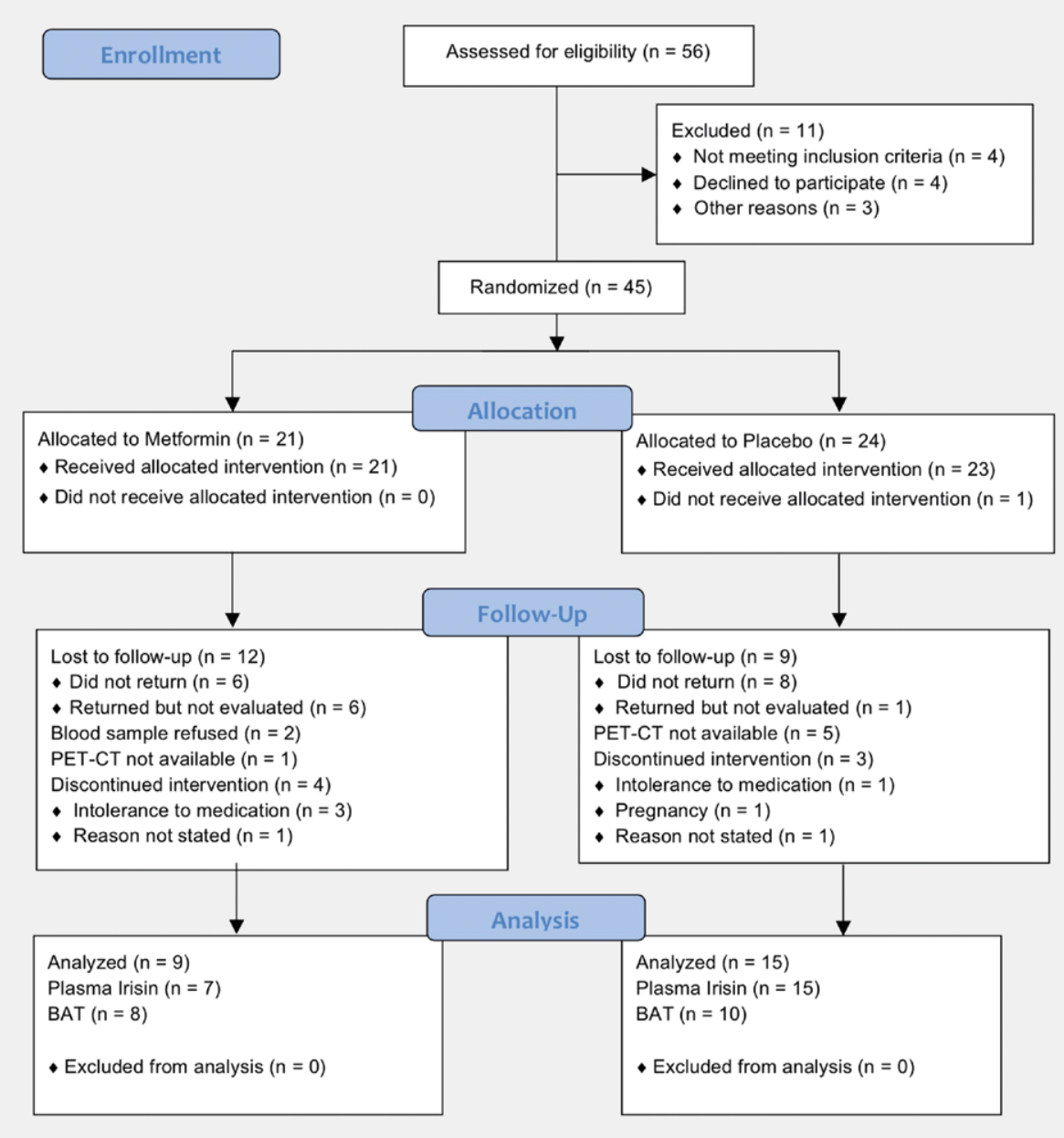

- Fig. 1 Flow diagram of the randomized trial of metformin versus placebo in women with PCOS.

\section{Results}

- Table 1 summarizes the baseline characteristics of the two study groups. The groups were similar in age, body measures, metabolic profile and PCOS phenotypes. The trial was completed by 9 participants of the metformin arm and by 15 participants of the placebo arm (> Fig. 1). Because our PET-CT facility had a period of shutdown, 6 participants that had completed the treatment course and returned to follow-up could not be reevaluated for BAT activity. Nevertheless, the anthropometric, clinical and biochemical characteristics of the metformin and placebo groups were similar at baseline, both comparing all randomized participants ( $\triangleright$ Table 1) and comparing only those who were available for follow-up (Supplemental $\triangleright$ Table 1S).

As shown in > Fig. 2, BAT activity did not change significantly after treatment with metformin (median $\Delta S U V_{\max }=-0.06 \mathrm{~g} / \mathrm{ml}$, interquartile interval -2.81 to $0.24 \mathrm{~g} / \mathrm{ml}, \mathrm{p}=0.484$, Wilcoxon's test) or placebo (median $\Delta S U V_{\max }=0.98 \mathrm{~g} / \mathrm{ml}$, interquartile interval -2.94 to $4.60 \mathrm{~g} / \mathrm{ml}, \mathrm{p}=0.386$ ). The median change of BAT activity did not differ significantly between the metformin and placebo groups ( $p=0.351$, Mann-Whitney test).

Plasma irisin levels remained essentially unchanged in the groups treated with metformin (median $\Delta=-98 \mathrm{ng} / \mathrm{ml}$, interquartile interval -366 to $60 \mathrm{ng} / \mathrm{ml}, \mathrm{p}=0.310$ ) and placebo (median
$\Delta=28 \mathrm{ng} / \mathrm{ml}$, interquartile interval -1260 to $215 \mathrm{ng} / \mathrm{ml}, \mathrm{p}=0.650$,

- Fig. 2d), although some participants had large differences between basal and 60 day plasma irisin levels regardless of being treated with metformin or placebo ( $\triangleright \mathbf{F i g} . \mathbf{2 c}$ ). The median change of plasma irisin levels activity did not differ significantly between the metformin and placebo groups ( $p=0.702$, Mann-Whitney test).

\section{Discussion}

In the present RCT, no significant change in BAT activity levels was observed from the beginning to the end of 60 days of treatment with either metformin or placebo. In a previous cross-sectional study, we had observed lower BAT activity in women with PCOS compared to healthy controls [9]. Our hypothesis was that metformin would improve insulin sensitivity and consequently rescue the normal BAT distribution in PCOS women. However, this hypothesis was not confirmed here. One possibility is that there was some improvement in insulin sensitivity with the metformin treatment but it was not sufficient to induce measurable changes in BAT activity. Early "browning” adipocytes (known as beige or brite) may not be evidenced by PET-CT since the cells that undergo the transdifferentiation process are immersed and dispersed in white fat sites [24]. In this context, high-resolution imaging coupled with 
a
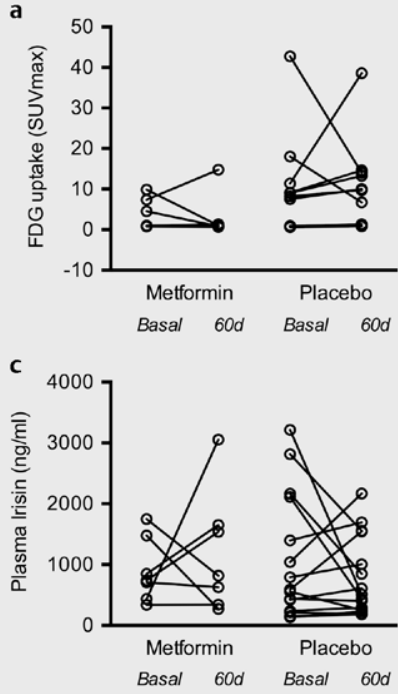

b

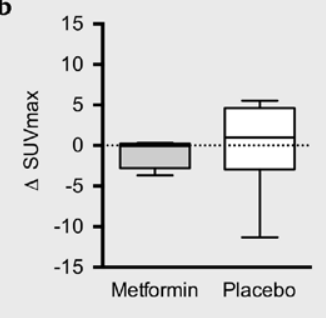

d

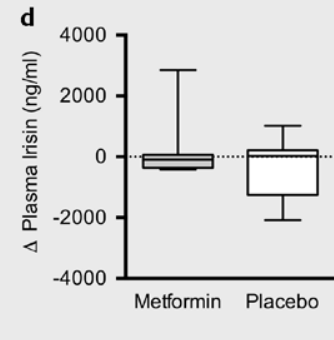

-Fig. 2 Individual plots (a, c) and median variations (b, d) of BAT activity and plasma irisin levels in PCOS women treated with metformin or placebo. The graphs represent the difference $(\Delta)$ between measures obtained before and after 60 days of treatment.

molecular characterization of tissue specimens may be needed to localize this early type of BAT [25]. In addition, we excluded women with glucose intolerance or diabetes, who are expected to benefit most from the use of metformin.

While the present study addressed the hypothesis that an insulin sensitizer (metformin) might increase BAT activity in humans, preclinical evidence suggests that interventions to increase the amount of BAT ultimately improve insulin sensitivity. Hu et al. [26] observed that three weeks of treatment with rutin, a novel compound for BAT activation, improved thermogenesis and systemic insulin sensitivity in a rat model of androgen-induced PCOS. Interestingly, BAT transplantation increased serum adiponectin and remarkably improved glucose homeostasis and insulin sensitivity in PCOS-like rats [27]. These translational results suggest that BAT activity is related to PCOS complications and that BAT induction might be a therapeutic option for PCOS treatment.

In this study, we observed no effect of metformin treatment for 60 days on plasma irisin levels in PCOS women. Our previous observations [9] as well as an extensive meta-analysis [28] indicated that plasma irisin levels are not altered in women with PCOS when compared with healthy controls of similar BMI. We decided to measure plasma irisin levels in the present study because irisin promotes UCP-1 expression and BAT differentiation with consequent improvement in glucose metabolism [15], therefore irisin could mediate any effect of metformin on BAT formation.

One possible reason for the lack of change in plasma irisin might be the relatively short treatment time, as a previous single-arm study of metformin treatment in PCOS women found a decrease in circulating irisin levels only at 6 months follow-up [29]. Another previous trial without a control treatment group administered metformin at low dose (500 mg once daily) during 3 months and observed a decrease of about $20 \%$ in plasma irisin levels [30]. These studies were open-label and had no control group, carrying the risk

of performance and detection biases. Our study had a double-blind, placebo-controlled design but it had a shorter intervention time and many participants were lost to follow-up. Therefore, further evidence from RCTs is still needed to clarify with a higher degree of confidence whether metformin treatment is able to change plasma irisin levels in women with PCOS.

As far as we know, this study is the first to evaluate the effect of metformin on BAT activity in humans. Our study achieved homogeneity between the metformin and placebo groups and was double blind to avoid the risk of selection, performance and detection biases. However, an important limitation of the present study is the smaller sample size at the end of the trial as a result of the participants lost to follow-up. While attrition bias is not evident by comparing the baseline characteristics of the participants that were fully analyzed, the statistical power of the trial was reduced. Consequently, further studies with a larger population are warranted.

In conclusion, no change in BAT activity and plasma irisin levels was observed after a brief treatment with metformin in women with PCOS.

\section{Funding}

Research supported by Fundação de Amparo à Pesquisa do Estado de Minas Gerais (FAPEMIG, grant \# APQ-03798-16) and Conselho Nacional de Desenvolvimento Científico e Tecnológico (CNPq) through the National Institute of Hormones and Women's Health and the National Institute of Molecular Medicine. K.B.G and F.M.R. received research grants from CNPq.

\section{Conflict of Interest}

The authors declare that they have no conflict of interest.

\section{References}

[1] Rocha AL, Oliveira FR, Azevedo RC et al. Recent advances in the understanding and management of polycystic ovary syndrome. F1000Res. 2019; 8: pii: F1000. Faculty Rev-565

[2] Azziz R, Kintziger K, Li R et al. Recommendations for epidemiologic and phenotypic research in polycystic ovary syndrome: An androgen excess and PCOS society resource. Hum Reprod 2019; 34: 2254-2265

[3] Teede HJ, Misso ML, Costello MF et al. Recommendations from the international evidence-based guideline for the assessment and management of polycystic ovary syndrome. Hum Reprod 2018; 33: 1602-1618

[4] Rothwell N], Stock M]. Effects of age on diet-induced thermogenesis and brown adipose tissue metabolism in the rat. Int J Obes 1983; 7: 583-589

[5] Gesta S, Tseng YH, Kahn CR. Developmental origin of fat: Tracking obesity to its source. Cell 2007; 131: 242-256

[6] van Marken Lichtenbelt WD, Vanhommerig JW, Smulders NM et al. Cold-activated brown adipose tissue in healthy men. $\mathrm{N}$ Engl J Med 2009; 360: 1500-1508

[7] Cypess AM, Lehman S, Williams $G$ et al. Identification and importance of brown adipose tissue in adult humans. N Engl J Med 2009; 360: 1509-1517 
[8] Nedergaard J, Bengtsson T, Cannon B. Unexpected evidence for active brown adipose tissue in adult humans. Am J Physiol Endocrinol Metab 2007; 293: E444-E452

[9] Oliveira FR, Mamede M, Bizzi MF et al. Brown adipose tissue activity is reduced in women with polycystic ovary syndrome. Eur J Endocrinol 2019; 181: 473-480

[10] Gourgari E, Spanakis E, Dobs AS. Pathophysiology, risk factors, and screening methods for prediabetes in women with polycystic ovary syndrome. Int J Womens Health 2016; 8: 381-387

[11] Spritzer PM. Polycystic ovary syndrome: Reviewing diagnosis and management of metabolic disturbances. Arq Bras Endocrinol Metabol 2014; 58: 182-187

[12] Wang C, Zhang XY, Sun $Y$ et al. Higher circulating irisin levels in patients with polycystic ovary syndrome: A meta-analysis. Gynecol Endocrinol 2018; 34: 290-293

[13] Breining P, Jensen JB, Sundelin El et al. Metformin targets brown adipose tissue in vivo and reduces oxygen consumption in vitro. Diabetes Obes Metab 2018; 20: 2264-2273

[14] Kim EK, Lee SH, Lee SY et al. Metformin ameliorates experimental-obesity-associated autoimmune arthritis by inducing FGF21 expression and brown adipocyte differentiation. Exp Mol Med 2018; 50: e432

[15] Bostrom P, Wu J, Jedrychowski MP et al. A PGC1-alpha-dependent myokine that drives brown-fat-like development of white fat and thermogenesis. Nature 2012; 481: 463-468

[16] Rotterdam EA-SPCWG Revised 2003 consensus on diagnostic criteria and long-term health risks related to polycystic ovary syndrome. Fertil Steril 2004; 81: 19-25

[17] Craig CL, Marshall AL, Sjostrom M et al. International physical activity questionnaire: 12-country reliability and validity. Med Sci Sports Exerc 2003; 35: 1381-1395

[18] Macfarlane DJ, Lee CC, Ho EY et al. Convergent validity of six methods to assess physical activity in daily life. J Appl Physiol 2006; 101: 1328-1334

[19] Colpani V, Oppermann K, Spritzer PM. Causes of death and associated risk factors among climacteric women from Southern Brazil: A population based-study. BMC Public Health 2014; 14: 194
[20] Bastos CA, Oppermann K, Fuchs SC et al. Determinants of ovarian volume in pre-, menopausal transition, and post-menopausal women: A population-based study. Maturitas 2006; 53: 405-412

[21] Sugawara Y, Zasadny KR, Neuhoff AW et al. Reevaluation of the standardized uptake value for FDG: Variations with body weight and methods for correction. Radiology 1999; 213: 521-525

[22] Nieuwenhuis-Ruifrok AE, Kuchenbecker WK, Hoek A et al. Insulin sensitizing drugs for weight loss in women of reproductive age who are overweight or obese: Systematic review and meta-analysis. Hum Reprod Update 2009; 15: 57-68

[23] Del Re AC, Maisel NC, Blodgett JC et al. Intention-to-treat analyses and missing data approaches in pharmacotherapy trials for alcohol use disorders. BMJ Open 2013; 3: e003464

[24] Wu J, Bostrom P, Sparks LM et al. Beige adipocytes are a distinct type of thermogenic fat cell in mouse and human. Cell 2012; 150: 366-376

[25] Lidell ME, Betz M], Dahlqvist Leinhard O et al. Evidence for two types of brown adipose tissue in humans. Nat Med 2013; 19: 631-634

[26] Hu T, Yuan X, Ye R et al. Brown adipose tissue activation by rutin ameliorates polycystic ovary syndrome in rat. J Nutr Biochem 2017; 47: $21-28$

[27] Yuan X, Hu T, Zhao H et al. Brown adipose tissue transplantation ameliorates polycystic ovary syndrome. Proc Natl Acad Sci USA 2016; 113: $2708-2713$

[28] Cai X, Qiu S, Li L et al. Circulating irisin in patients with polycystic ovary syndrome: A meta-analysis. Reprod Biomed Online 2018; 36: $172-180$

[29] Li M, Yang M, Zhou X et al. Elevated circulating levels of irisin and the effect of metformin treatment in women with polycystic ovary syndrome. J Clin Endocrinol Metab 2015; 100: 1485-1493

[30] Masaeli A, Nayeri H, Mirzaee M. Effect of metformin treatment on insulin resistance markers, and circulating irisin in women with Polycystic Ovarian Syndrome (PCOS). Horm Metab Res 2019; 51 575-579 